Review Article

\title{
Submucosal Injection Solution for Endoscopic Resection in Gastrointestinal Tract: A Traditional and Network Meta-Analysis
}

\author{
Zhang Yu Huai, Wei Feng Xian, Luo Chang Jiang, and Wang Xi Chen \\ Department of General Surgery, Lanzhou University Second Hospital, Lanzhou 730030, China \\ Correspondence should be addressed to Zhang Yu Huai; zhang62968@sina.com
}

Received 26 October 2014; Revised 5 December 2014; Accepted 5 December 2014

Academic Editor: Peter V. Draganov

Copyright ( 2015 Zhang Yu Huai et al. This is an open access article distributed under the Creative Commons Attribution License, which permits unrestricted use, distribution, and reproduction in any medium, provided the original work is properly cited.

\begin{abstract}
Objective. To explore and define the current optimal submucosal injection solution used in ESD and EMR for gastrointestinal tract neoplasms in terms of clinical outcomes and other aspects. Methods. PubMed, Cochrane Library, Embase, and clinical trials register center were searched with terms of "endoscopic resection" and "submucosal injection solution" to identify relevant randomized controlled trials (RCTs). Both direct comparison using traditional meta-analysis method and indirect comparison using network meta-analysis method were performed. Results. A total of 11 RCTs with 1152 patients were included. Meta-analysis showed that, compared with normal saline, other submucosal injection solutions induced a significant increase in terms of en bloc resection rate $\left(I^{2}=0 \%, \mathrm{OR}=2.11,95 \% \mathrm{CI}(1.36,3.26)\right.$, and $\left.P=0.008\right)$ and complete resection rate $\left(I^{2}=0 \%, \mathrm{OR}=2.14,95 \% \mathrm{CI}(1.41,3.24)\right.$, and $P=0.0003)$; and there was no significant difference in the incidence of total complications $\left(I^{2}=0 \%, \mathrm{OR}=0.87,95 \% \mathrm{CI}(0.59\right.$, 1.29), and $P=0.49$ ). Conclusions. Other newly developed submucosal injection solutions significantly increased en bloc resection rate and complete resection rate and decreased bleeding rate and finical cost of endoscopic resection in gastrointestinal tract, while current evidence did not find the difference between them, which need to be explored by further studies.
\end{abstract}

\section{Introduction}

With the diagnosis technique and accuracy of early gastrointestinal (GI) tract cancer increased, endoscopic treatment is widely applied as a radical curative therapy [1]. Among kinds of operations, endoscopic mucosal resection (EMR) and endoscopic submucosal dissection (ESD) are the most frequently used as they are minimally invasive especially for sessile and flat polyps [2]. They are proposed as replacements for invasive surgery in early gastrointestinal neoplasm (with low risk of lymph-node metastasis) due to simplified procedure, decreased cost, improved quality of life, and favorable long-term benefit $[3,4]$.

ESD overcomes the limitation that when a lesion is larger than $20 \mathrm{~mm}$, EMR could hardly enable en bloc resection, while compared with EMR it increases the operation time and the risk of complications such as bleeding and perforation [5]. In order to facilitate easier and safe performance of the two procedures, a submucosal injection solution is always required to lift the lesion from muscular layer. An optimal injection solution must primarily contribute to achievement of better clinical outcomes and meanwhile should firstly achieve and maintain the necessary submucosal lifting height and duration [6], secondly not influence the histological evaluation, thirdly not have tissue toxicity [7], and fourthly be easily prepared and administrated with low cost. Currently, various submucosal injection solutions including normal saline (NS), fibrinogen mixture (FM), dextrose (DE), glycerol, sodium hyaluronic acid (SHA), succinylated gelatin (SG), hydroxyethyl starch (HES), and mesna (ME) are developed. And many studies have compared the various injections in EMR and ESD for GI tract neoplasm in terms of clinical efficacy and safety.

However, the results of these studies are not completely consistent, and the number of direct comparison studies is not sufficient. So, in order to explore and define the current optimal submucosal injection solution used in ESD and EMR for GI tract neoplasm in terms of clinical outcomes, we performed this meta-analysis of all eligible randomized controlled trials. 


\section{Materials and Methods}

2.1. Literature Search. A systematic search was performed on databases including PubMed (1966.01-2014.07), Cochrane Library (2014 Issue 07), Embase (1974.01-2014.07), and clinical trials register center (up to 2014.07). Search terms were ("endoscopic submucosal dissection" OR "ESD" OR “endoscopic mucosal resection" OR "EMR" OR “endoscopic resection") AND ("submucosal injection solution" OR "hyaluronic acid” OR "normal Saline” OR “dextrose”). Medical subject headings and extend function were also used to identify all relevant studies including abstracts, comments, reviews, clinical studies, and citations.

2.2. Inclusion and Exclusion Criteria. Only randomized controlled trials (RCTs) published in English were eligible. Patients with gastrointestinal tract lesions who were willing to receive polypectomy were participants, and they were randomized to treatment group or control group. All the interventions were comparable between the two groups except for the submucosal injection solution used. The main outcome measures to evaluate therapeutic efficacy were en bloc resection (defined as resection without piecemeal separation) rate and complete resection (defined as absence of neoplastic tissue in the edge of the cut lesion) rate. Secondary outcome measures were the incidence of total complications, bleeding, perforation, and recurrence. The primary literature search results were scanned by abstracts and further assessment was reading the full-text by two reviewers independently.

2.3. Data Extraction and Quality Assessment. Two reviewers extracted basic information (such as the first author, publication year, group, and case) and data (main and secondary outcome measures) from the included studies. Methodological quality was assessed according to the method recommended by the Cochrane Handbook [8], mainly based on six items: randomization, allocation concealment, blinding, comparative baseline, $>80 \%$ follow-up, and freedom of selective reporting. According to quality assessment results, studies were judged as levels A (all the six items were appropriate), B (four or five items were appropriate), and C (less than four items were appropriate). And any disagreements about eligibility, data, and quality assessment were resolved through discussion.

2.4. Statistical Analysis. We entered all the extracted data into a predesigned table and did traditional meta-analysis using Revman software (version 5.29, recommended by the Cochrane Collaboration). Chi-square and $I^{2}$ statistic were adopted to assess the heterogeneity between trials. Subgroup analysis was performed to explore the specific clinical outcomes and complications, and funnel plot was used to evaluate the potential risk of publication bias. Then pooled odds ratios (OR) and their 95\% confidence intervals (95\% CI) were summarized and used for network metaanalysis. We did network meta-analysis using ITC software (version 1.0, Canadian Agency for Drugs and Technologies in Health, Indirect Treatment Comparison Software, Ottawa,

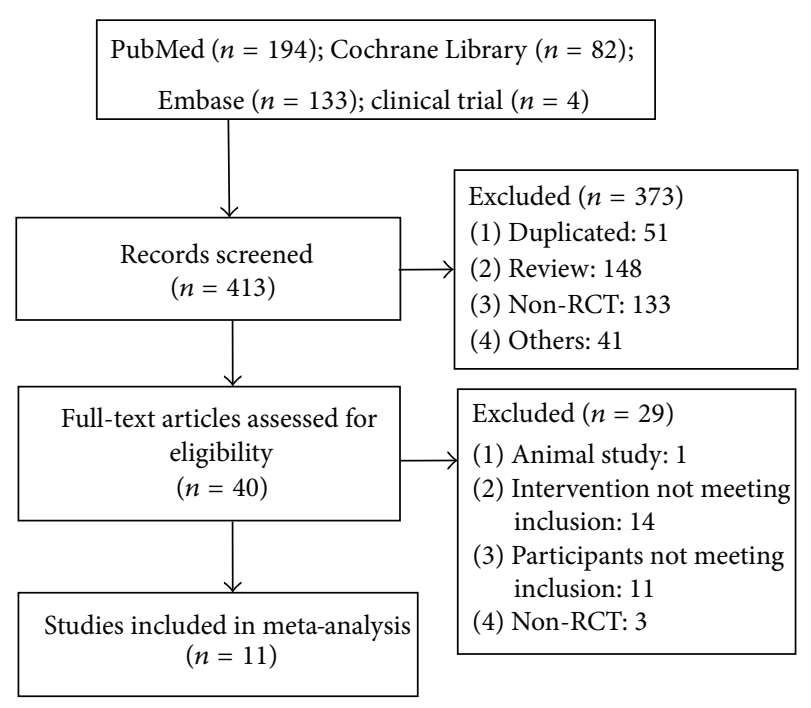

FIgURE 1: Flow diagram of trial selection.

Ontario, Canada) [9]. The head-to-head indirect comparison was handled and then assigned a result in terms of statistical superiority/inferiority or no difference between the groups, and relevant forest plots were also presented.

\section{Results}

3.1. Flow Diagram of Trial Selection. In total, eleven RCTs [10-20] containing 1152 patients were included. Figure 1 shows a flow diagram of trial selection from the initial search result to the final inclusion. The basic information about the publications, the participants, and the lesions was described in Table 1. The methodological method assessment showed that 7 RCTs reached a level of A [10-12, 15, 16, 19, 20], 2 RCTs reached a level of $\mathrm{B}[13,17]$, and 2 RCTs reached a level of $\mathrm{C}$ $[14,18]$ (Table 2).

\subsection{Direct Comparison of Submucosal Injection Solution with NS}

3.2.1. Pooled Analysis. Compared with normal saline (NS), other submucosal injection solutions used in ESD or EMR for gastrointestinal lesions induced a significant increase in terms of en bloc resection rate $\left(I^{2}=0 \%, \mathrm{OR}=2.11,95 \% \mathrm{CI}(1.36\right.$, 3.26), and $P=0.008$, Figure 3 ) and complete resection rate $\left(I^{2}=0 \%\right.$, OR $=2.14,95 \%$ CI $(1.41,3.24)$, and $P=0.0003$, Figure 4). Though there was no significant difference in the incidence of total complications $\left(I^{2}=0 \%\right.$, OR $=0.87,95 \%$ CI $(0.59,1.29)$, and $P=0.49$, Figure 5).

3.2.2. Subgroup Analysis. Subgroup analysis indicated that, compared with NS, other submucosal injection solutions significantly increased en bloc resection rate $\left(I^{2}=0 \%, \mathrm{OR}=\right.$ $0.62,95 \%$ CI $(0.39,0.98)$, and $P=0.04)$ and complete resection rate $\left(I^{2}=0 \%, \mathrm{OR}=0.62,95 \% \mathrm{CI}(0.39,0.98)\right.$, and $P=$ 0.04 ) in EMR subgroup, as shown in Figure 3. Compared with 
TABLE 1: Characteristics of participants.

\begin{tabular}{|c|c|c|c|c|c|c|c|c|}
\hline Study & Group & Case & Age & $\begin{array}{c}\text { Sex } \\
(\mathrm{M} / \mathrm{F})\end{array}$ & $\begin{array}{l}\text { Size } \\
(\mathrm{mm})\end{array}$ & Site & Method & Type \\
\hline \multirow{2}{*}{$\begin{array}{l}\text { Lee et al., } \\
2006[10]\end{array}$} & Fibrinogen & 36 & $61.4 \pm 11.5$ & $27 / 9$ & $19.2 \pm 6.3$ & $\begin{array}{l}\text { Gastric, proximal 2/3 (11), } \\
\text { distal 1/3 (25) }\end{array}$ & \multirow{2}{*}{ EMR } & $\begin{array}{l}\text { Adenoma (24), } \\
\text { adenocarcinoma (12) }\end{array}$ \\
\hline & NS & 36 & $59.7 \pm 11.2$ & $21 / 15$ & $16.8 \pm 6.1$ & $\begin{array}{l}\text { Gastric, proximal 2/3 (12), } \\
\text { distal } 1 / 3(24)\end{array}$ & & $\begin{array}{l}\text { Adenoma (25), } \\
\text { adenocarcinoma (11) }\end{array}$ \\
\hline \multirow{2}{*}{$\begin{array}{l}\text { Hurlstone et } \\
\text { al., } 2008[11]\end{array}$} & Dextrose & 82 & $56(29-84)^{*}$ & $42 / 40$ & $18(6-35)^{*}$ & Colon, left (33), right (49) & \multirow{2}{*}{ EMR } & $\begin{array}{l}\text { LGD (58), HGD (19), } \\
\text { carcinoma (5) }\end{array}$ \\
\hline & $0.4 \%$ SHA & 81 & $58(32-83)^{*}$ & $39 / 42$ & $20(4-40)^{*}$ & Colon, left (36), right (45) & & $\begin{array}{l}\text { LGD (59), HGD (18), } \\
\text { carcinoma (4) }\end{array}$ \\
\hline \multirow{2}{*}{$\begin{array}{l}\text { Katsinelos et } \\
\text { al., } 2008 \text { [12] }\end{array}$} & Dextrose & 45 & $65(42-82)^{*}$ & $20 / 25$ & \multirow[t]{2}{*}{$>10$} & $\begin{array}{l}\text { Rectosigmoid, rectum } \\
(27), \text { sigmoid (18) }\end{array}$ & \multirow[t]{2}{*}{ EMR } & $\begin{array}{l}\text { LGD (16), MGD (18), } \\
\text { HGD (9), carcinoma (2) }\end{array}$ \\
\hline & NS & 47 & $69(41-92)^{*}$ & $27 / 20$ & & $\begin{array}{l}\text { Rectosigmoid, rectum } \\
(27), \text { sigmoid (20) }\end{array}$ & & $\begin{array}{l}\text { LGD (23), MGD (13), } \\
\text { HGD (10), carcinoma (1) }\end{array}$ \\
\hline \multirow{2}{*}{$\begin{array}{l}\text { Yamamoto et } \\
\text { al., } 2008 \text { [13] }\end{array}$} & $0.4 \%$ SHA & 69 & $65.3 \pm 8.1$ & $57 / 12$ & \multirow{2}{*}{$5-20^{*}$} & $\begin{array}{l}\text { Gastric, proximal (8), } \\
\text { body (30), distal (31) }\end{array}$ & \multirow{2}{*}{ ESD \& EMR } & $\begin{array}{l}\text { Adenoma (18), } \\
\text { adenocarcinoma (58) }\end{array}$ \\
\hline & NS & 70 & $66.1 \pm 8.4$ & $51 / 19$ & & $\begin{array}{l}\text { Gastric, proximal (8), } \\
\text { body (28), distal (34) }\end{array}$ & & $\begin{array}{l}\text { Adenoma (17), } \\
\text { adenocarcinoma (53) }\end{array}$ \\
\hline \multirow{2}{*}{$\begin{array}{l}\text { Kizu et al., } \\
2010[14]\end{array}$} & $0.4 \%$ SHA & 53 & \multirow[t]{2}{*}{-} & \multirow[t]{2}{*}{-} & \multirow{2}{*}{$<20$} & \multirow{2}{*}{ Gastric } & \multirow{2}{*}{ ESD } & \multirow{2}{*}{-} \\
\hline & NS & 52 & & & & & & \\
\hline \multirow{2}{*}{$\begin{array}{l}\text { Moss et al., } \\
2010[15]\end{array}$} & SG & 41 & $69(64-76)^{\#}$ & $22 / 19$ & $40(25-45)^{\#}$ & $\begin{array}{l}\text { Colon, proximal to } \\
\text { hepatic flexure (17) }\end{array}$ & \multirow{2}{*}{ EMR } & $\begin{array}{l}\text { Tubular (18), } \\
\text { tubulovillous (16), sessile } \\
\text { serrated adenoma (7) }\end{array}$ \\
\hline & NS & 39 & $67(62-78)^{\#}$ & $23 / 16$ & $35(30-50)^{\#}$ & $\begin{array}{l}\text { Colon, proximal to } \\
\text { hepatic flexure (14) }\end{array}$ & & $\begin{array}{l}\text { Tubular (11), } \\
\text { tubulovillous (22), sessile } \\
\text { serrated adenoma (6) }\end{array}$ \\
\hline \multirow{2}{*}{$\begin{array}{l}\text { Fasoulas et } \\
\text { al., } 2012[16]\end{array}$} & HES & 25 & $68(43-82)^{*}$ & $16 / 9$ & $4.5(3.2-7)^{*}$ & $\begin{array}{l}\text { Colorectal, rectum (19), } \\
\text { ascending colon (3), } \\
\text { cecum (3) }\end{array}$ & \multirow{2}{*}{ EMR } & $\begin{array}{l}\text { Paris classification, O-II } \\
\text { a (19), O-II b (6) }\end{array}$ \\
\hline & NS & 24 & $67(48-88)^{*}$ & $8 / 16$ & $4.6(3.3-7.2)^{*}$ & $\begin{array}{l}\text { Colorectal, rectum (18), } \\
\text { ascending colon ( } 3), \\
\text { cecum }(3)\end{array}$ & & $\begin{array}{l}\text { Paris classification, O-II } \\
\text { a (21), O-II b (3) }\end{array}$ \\
\hline \multirow{2}{*}{$\begin{array}{l}\text { Kishihara et } \\
\text { al., } 2012 \text { [17] }\end{array}$} & $0.2 \%$ SHA & 46 & $61.0 \pm 9.0$ & $21 / 25$ & $11.3 \pm 3.0$ & $\begin{array}{l}\text { Colorectal, proximal (20), } \\
\text { distal (22), rectum (4) }\end{array}$ & \multirow{2}{*}{ EMR } & $\begin{array}{l}\text { Adenoma (39), } \\
\text { adenocarcinoma (7) }\end{array}$ \\
\hline & NS & 48 & $65.0 \pm 8.0$ & $32 / 16$ & $12.5 \pm 4.0$ & $\begin{array}{l}\text { Colorectal, proximal (26), } \\
\text { distal (16), rectum (6) }\end{array}$ & & $\begin{array}{l}\text { Adenoma (40), } \\
\text { adenocarcinoma (8) }\end{array}$ \\
\hline \multirow{2}{*}{$\begin{array}{l}\text { Yoshida et al., } \\
2012[18]\end{array}$} & $0.13 \%$ SHA & 93 & $66(23-85)^{*}$ & $62 / 31$ & $8.9(8-16)^{*}$ & $\begin{array}{l}\text { Colorectal, cecum to } \\
\text { descending colon (49), } \\
\text { rectum to sigmoid (44) }\end{array}$ & \multirow[t]{2}{*}{ EMR } & $\begin{array}{l}\text { Adenoma (84), } \\
\text { adenocarcinoma (9) }\end{array}$ \\
\hline & NS & 96 & $67(35-89)^{*}$ & $63 / 33$ & $8.2(5-15)^{*}$ & $\begin{array}{l}\text { Colorectal, cecum to } \\
\text { descending colon (50), } \\
\text { rectum to sigmoid (46) }\end{array}$ & & $\begin{array}{l}\text { Adenoma (92), } \\
\text { adenocarcinoma (4) }\end{array}$ \\
\hline \multirow{2}{*}{$\begin{array}{l}\text { Kim et al., } \\
2013 \text { [19] }\end{array}$} & $0.4 \%$ SHA & 29 & $62.6 \pm 9.2$ & $25 / 12$ & $14.2 \pm 5.47$ & $\begin{array}{l}\text { Gastric, antrum (23), } \\
\text { angle (3), body (11) }\end{array}$ & \multirow{2}{*}{ ESD } & $\begin{array}{l}\text { Adenoma (31), atypia (1), } \\
\text { adenocarcinoma (5) }\end{array}$ \\
\hline & NS & 34 & $62.4 \pm 9.9$ & $26 / 13$ & $13.5 \pm 4.35$ & $\begin{array}{l}\text { Gastric, antrum (29), } \\
\text { angle (3), body ( } 7)\end{array}$ & & $\begin{array}{l}\text { Adenoma (31), atypia (1), } \\
\text { adenocarcinoma (7) }\end{array}$ \\
\hline \multirow{2}{*}{$\begin{array}{l}\text { Sumiyama et } \\
\text { al., } 2014[20]\end{array}$} & Mesna & 53 & - & $41 / 9$ & $19.5 \pm 11.5$ & $\begin{array}{l}\text { Gastric, upper }(7), \text { middle } \\
(25), \text { lower }(21)\end{array}$ & \multirow[t]{2}{*}{ ESD } & $\begin{array}{l}\text { Adenoma (7), } \\
\text { adenocarcinoma (46) }\end{array}$ \\
\hline & NS & 53 & - & $41 / 9$ & $17.1 \pm 10.1$ & $\begin{array}{l}\text { Gastric, upper (12), } \\
\text { middle (20), lower (20) }\end{array}$ & & $\begin{array}{l}\text { Adenoma (5), } \\
\text { adenocarcinoma (47) }\end{array}$ \\
\hline
\end{tabular}

E, epinephrine; HES, hydroxyethyl starch; SG, succinylated gelatin.

${ }^{*}$ Median (range), ${ }^{*}$ median (interquartile range).

LGD, low-grade dysplasia; MGD, moderate-grade dysplasia; HGD, high-grade dysplasia. 
TABLE 2: Quality assessment of included randomized controlled trials.

\begin{tabular}{|c|c|c|c|c|c|c|c|c|}
\hline Study & State & Randomization & $\begin{array}{c}\text { Allocation } \\
\text { concealment }\end{array}$ & Blinding & $\begin{array}{l}\text { Comparable } \\
\text { baseline }\end{array}$ & $\begin{array}{l}>80 \% \\
\text { follow-up }\end{array}$ & $\begin{array}{l}\text { Freedom of } \\
\text { selective } \\
\text { reporting }\end{array}$ & Level \\
\hline $\begin{array}{l}\text { Lee et al., } \\
2006[10]\end{array}$ & Korea & $\begin{array}{l}\mathrm{Y} \text {, central controlled } \\
\text { randomization }\end{array}$ & $\mathrm{Y}$ & $\begin{array}{l}\text { Y, double } \\
\text { blind }\end{array}$ & $\mathrm{Y}$ & $\mathrm{Y}$ & $\mathrm{Y}$ & A \\
\hline $\begin{array}{l}\text { Hurlstone et } \\
\text { al., } 2008 \text { [11] }\end{array}$ & UK & $\mathrm{Y}$, random sequence & $\mathrm{Y}$ & $\begin{array}{l}\text { Y, double } \\
\text { blind }\end{array}$ & $\mathrm{Y}$ & $\mathrm{Y}$ & $\mathrm{Y}$ & A \\
\hline $\begin{array}{l}\text { Katsinelos et } \\
\text { al., } 2008 \text { [12] }\end{array}$ & Greece & $\mathrm{Y}$, random number & $\mathrm{Y}$ & $\begin{array}{l}\text { Y, double } \\
\text { blind }\end{array}$ & $\mathrm{Y}$ & $\mathrm{Y}$ & $\mathrm{Y}$ & A \\
\hline $\begin{array}{l}\text { Yamamoto et } \\
\text { al., } 2008 \text { [13] }\end{array}$ & Japan & $\begin{array}{l}\mathrm{Y} \text {, center controlled } \\
\text { randomization }\end{array}$ & Unclear & Unclear & $\mathrm{Y}$ & $\mathrm{Y}$ & $\mathrm{Y}$ & B \\
\hline $\begin{array}{l}\text { Kizu et al., } \\
2010[14]\end{array}$ & - & M & Unclear & Unclear & $\mathrm{Y}$ & $\mathrm{Y}$ & Unclear & $\mathrm{C}$ \\
\hline $\begin{array}{l}\text { Moss et al., } \\
2010[15]\end{array}$ & Australia & $\mathrm{Y}$, random sequence & $\mathrm{Y}$ & $\begin{array}{l}\text { Y, double } \\
\text { blind }\end{array}$ & $\mathrm{Y}$ & $\mathrm{Y}$ & $\mathrm{Y}$ & A \\
\hline $\begin{array}{l}\text { Fasoulas et } \\
\text { al., } 2012[16]\end{array}$ & Greece & $\begin{array}{l}\mathrm{Y}, \text { block balance } \\
\text { random sequence }\end{array}$ & $\mathrm{Y}$ & $\begin{array}{l}\text { Y, double } \\
\text { blind }\end{array}$ & $\mathrm{Y}$ & $\mathrm{Y}$ & $\mathrm{Y}$ & A \\
\hline $\begin{array}{l}\text { Kishihara et } \\
\text { al., } 2012 \text { [17] }\end{array}$ & Japan & M & $\mathrm{Y}$ & Unclear & $\mathrm{Y}$ & $\mathrm{Y}$ & $\mathrm{Y}$ & B \\
\hline $\begin{array}{l}\text { Yoshida et al., } \\
2012 \text { [18] }\end{array}$ & Japan & M & Unclear & $\mathrm{N}$ & Y & $\mathrm{Y}$ & $\mathrm{Y}$ & $\mathrm{C}$ \\
\hline $\begin{array}{l}\text { Kim et al., } \\
2013 \text { [19] }\end{array}$ & Korea & $\mathrm{Y}$, random sequence & $\mathrm{Y}$ & $\begin{array}{l}\text { Y, double } \\
\text { blind }\end{array}$ & $\mathrm{Y}$ & $\mathrm{Y}$ & $\mathrm{Y}$ & $\mathrm{A}$ \\
\hline $\begin{array}{l}\text { Sumiyama et } \\
\text { al., } 2014[20]\end{array}$ & Japan & $\begin{array}{l}\text { Y, computer generated } \\
\text { random sequence }\end{array}$ & $\mathrm{Y}$ & $\begin{array}{l}\text { Y, double } \\
\text { blind }\end{array}$ & $\mathrm{Y}$ & $\mathrm{Y}$ & $\mathrm{Y}$ & A \\
\hline
\end{tabular}

$\mathrm{M}$, the method was mentioned, but there was not a detailed description; $\mathrm{Y}$, the method was reported with detailed description; Unclear, no relevant information was found in the study.

NS, other submucosal injections also significantly increased en bloc resection rate $\left(I^{2}=0 \%, \mathrm{OR}=0.62,95 \% \mathrm{CI}(0.39,0.98)\right.$, and $P=0.04)$ and complete resection rate $\left(I^{2}=0 \%, \mathrm{OR}=\right.$ $0.62,95 \% \mathrm{CI}(0.39,0.98)$, and $P=0.04)$ in ESD subgroup, as shown in Figure 4.

Subgroup analysis of complications indicated that, compared with NS, other submucosal injections significantly decreased the incidence of bleeding $\left(I^{2}=0 \%, \mathrm{OR}=0.62,95 \%\right.$ CI $(0.39,0.98)$, and $P=0.04)$, while significantly increasing the incidence of postpolypectomy syndrome $\left(I^{2}=0 \%\right.$, OR $=6.42,95 \% \mathrm{CI}(1.10,37.62)$, and $P=0.04)$, and there was no significant difference in terms of perforation $\left(I^{2}=0 \%\right.$, OR $=0.96,95 \%$ CI $(0.19,4.87)$, and $P=0.96)$ and recurrence $\left(I^{2}=0 \%, \mathrm{OR}=0.56,95 \% \mathrm{CI}(0.23,1.38)\right.$, and $\left.P=0.21\right)$.

\subsection{Indirect Comparison of Submucosal Injection Solutions.} Figure 2 shows the network of clinical trials according to the specific classes of initial injected solutions. Indirect comparison using network meta-analysis method according to the solution classes was mainly conducted in en bloc resection rate and complete resection rate, as shown in Table 3.

3.3.1. En Bloc Resection Rate. The OR value for fibrinogen ranged from 0.18 to 0.43 compared with the others, and there was a significant difference between fibrinogen and dextrose

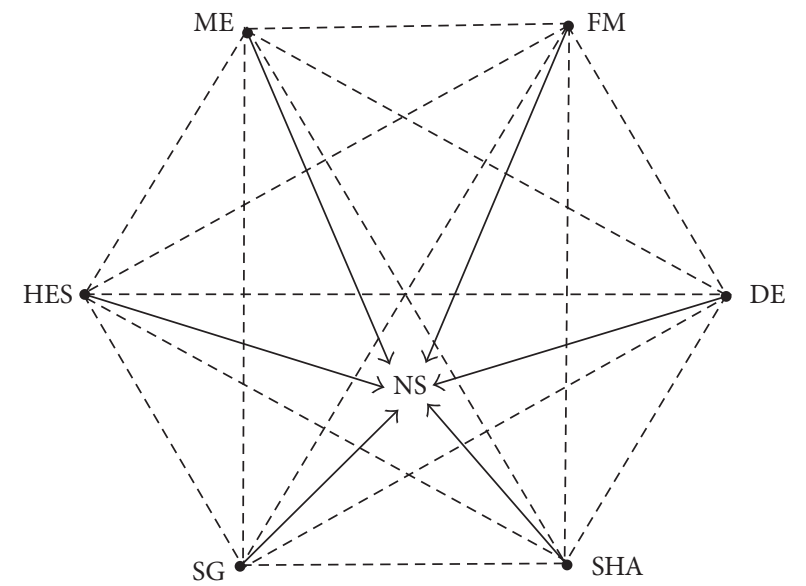

FIgURE 2: Network of clinical trials according to the comparison of specific classes of initial injected solutions. NS, normal saline; FM, fibrinogen mixture; DE, dextrose; SHA, sodium hyaluronic acid; SG, succinylated gelatin; HES, hydroxyethyl starch; and ME, mesna.

$(\mathrm{OR}=0.18,95 \% \mathrm{CI}(0.04,0.89))$. The OR value for dextrose ranged from 1.26 to 2.39 compared with the others, while the difference failed to reach any statistical significance. The OR value for hyaluronate acid ranged from 0.85 to 1.62 compared with the others, and there was no significant difference. 


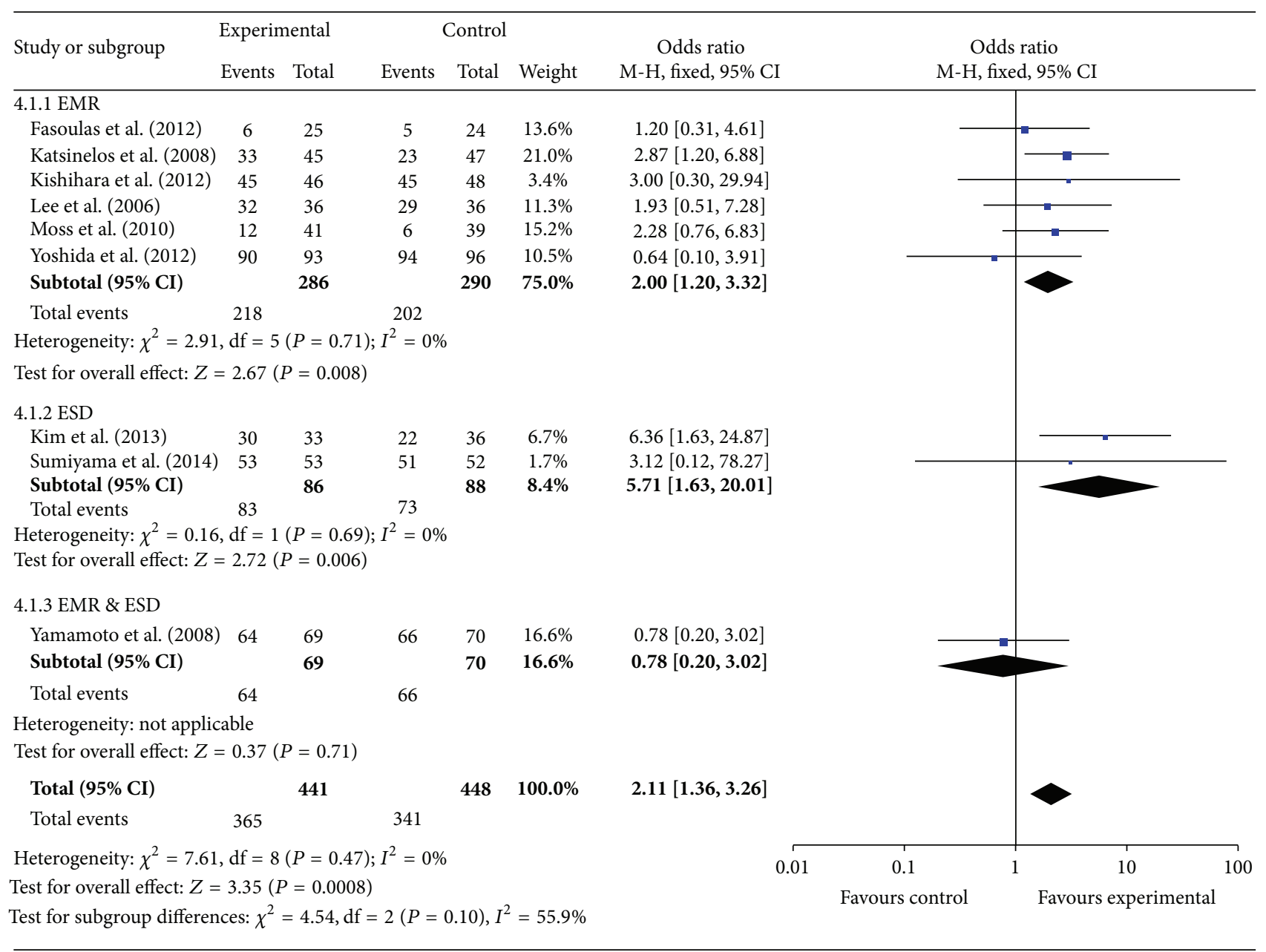

FIGURE 3: Meta-analysis results of en bloc resection rate between the two groups.

The difference between succinylated gelatin and hydroxyethyl starch also failed to reach statistical significance.

3.3.2. Complete Resection Rate. The OR value for fibrinogen ranged from 0.48 to 1.44 compared with the others, and there was no significant difference. The OR value for dextrose ranged from 0.66 to 1.97 compared with the others, and the difference failed to reach statistical significance. The OR value for hyaluronate acid ranged from 0.77 to 2.31 compared with the others, and there was no significant difference. The differences among succinylated gelatin, hydroxyethyl starch, and mesna also failed to reach statistical significance.

3.4. Publication Bias. Funnel plots were adopted to evaluate the publication bias. The shapes of funnel plots for en bloc resection rate, complete resection rate, and total complications did not reveal asymmetry, indicating no evidence of publication bias (Figure 6).

\section{Discussion}

This meta-analysis was the first to explore the influence of different classes of submucosal injection solutions for endoscopic resection and found a distinct advantage of other submucosal injection solutions than NS. The en bloc resection rate $(82 \%$ versus $77 \%)$ and complete resection rate $(89 \%$ versus $79 \%$ ) were both significantly higher in the other submucosal injection solution group. Though there was no significant difference in the incidence of total complications, subgroup analysis indicated that other injection solutions significantly decreased the incidence of bleeding, while some of them increased the incidence of postpolypectomy syndrome. There was no significant difference between the two groups in terms of recurrence and perforation. Heterogeneity test and inverted funnel plot demonstrated that there were little heterogeneity and publication bias, and all the results were pooled in the fixed-effect models.

As known, EMR was mostly adopted for lesion size smaller than $20 \mathrm{~mm}$, and ESD was mostly applied for lesion size larger than 20-30 mm with increased technical difficulties and equipment requirement [21]. Of the included studies, 6 applied EMR, 4 applied ESD, and 1 applied ESD and EMR according the lesion size. A previous meta-analysis demonstrated that there was no significant difference between ESD and EMR in terms of en bloc resection rate and complete resection rate, and ESD was only associated with prolonged 


\begin{tabular}{|c|c|c|c|c|c|c|c|c|c|c|}
\hline \multirow{3}{*}{$\begin{array}{l}\text { Study or subgroup } \\
4.2 .1 \mathrm{EMR}\end{array}$} & \multicolumn{2}{|c|}{ Experimental } & \multicolumn{3}{|c|}{ Control } & \multirow{2}{*}{$\begin{array}{c}\text { Odds ratio } \\
\text { M-H, fixed, } 95 \% \text { CI }\end{array}$} & \multirow{2}{*}{\multicolumn{4}{|c|}{$\begin{array}{c}\text { Odds ratio } \\
\text { M-H, fixed, 95\% CI }\end{array}$}} \\
\hline & \multirow[t]{2}{*}{ Events } & \multirow[t]{2}{*}{ Total } & \multirow[t]{2}{*}{ Events } & \multirow[t]{2}{*}{ Total } & \multirow{2}{*}{ Weight } & & & & & \\
\hline & & & & & & & & & & \\
\hline Fasoulas et al. (2012) & 24 & 25 & 23 & 24 & $3.0 \%$ & $1.04[0.06,17.69]$ & & & & \\
\hline Katsinelos et al. (2008) & 42 & 45 & 41 & 47 & $8.5 \%$ & $2.05[0.48,8.74]$ & & & & \\
\hline Kizu et al. (2010) & 45 & 53 & 39 & 52 & $19.0 \%$ & $1.88[0.70,4.99]$ & & & & \\
\hline Lee et al. (2006) & 32 & 36 & 29 & 36 & $10.3 \%$ & $1.93[0.51,7.28]$ & & & 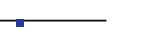 & \\
\hline Moss et al. (2010) & 37 & 41 & 35 & 39 & $11.2 \%$ & $1.06[0.25,4.56]$ & & & & \\
\hline Yoshida et al. (2012) & 74 & 93 & 63 & 96 & $40.4 \%$ & $2.04[1.06,3.93]$ & & & & \\
\hline Subtotal $(95 \% \mathrm{CI})$ & & 293 & & 294 & $92.4 \%$ & $1.84[1.18,2.88]$ & & & & \\
\hline Total events & 254 & & 230 & & & & & & & \\
\hline \multicolumn{11}{|c|}{ Heterogeneity: $\chi^{2}=0.83, \mathrm{df}=5(P=0.98) ; I^{2}=0 \%$} \\
\hline \multicolumn{11}{|c|}{ Test for overall effect: $Z=2.69(P=0.007)$} \\
\hline \multicolumn{11}{|l|}{ 4.2.2 ESD } \\
\hline Kim et al. (2013) & 30 & 33 & 22 & 36 & $6.1 \%$ & $6.36[1.63,24.87]$ & & & & \\
\hline Sumiyama et al. (2014) & 53 & 53 & 51 & 52 & $1.5 \%$ & $3.12[0.12,78.27]$ & & & & \\
\hline Subtotal $(95 \% \mathrm{CI})$ & & 86 & & 88 & $7.6 \%$ & $5.71[1.63,20.01]$ & & & & \\
\hline Total events & 83 & & 73 & & & & & & & \\
\hline \multicolumn{11}{|c|}{ Heterogeneity: $\chi^{2}=0.16, \mathrm{df}=1(P=0.69) ; I^{2}=0 \%$} \\
\hline \multicolumn{11}{|c|}{ Test for overall effect: $Z=2.72(P=0.006)$} \\
\hline Total $(95 \% \mathrm{CI})$ & & 379 & & 382 & $100.0 \%$ & $2.14[1.41,3.24]$ & & & & \\
\hline Total events & 337 & & 303 & & & & & & & \\
\hline \multicolumn{6}{|c|}{ Heterogeneity: $\chi^{2}=3.77, \mathrm{df}=7(P=0.81) ; I^{2}=0 \%$} & 0.01 & 0.1 & 1 & 10 & 100 \\
\hline \multicolumn{7}{|c|}{ Test for overall effect: $Z=3.59(P=0.0003)$} & Favours control & & Favours expe & \\
\hline \multicolumn{11}{|c|}{ Test for subgroup differences: $\chi^{2}=2.77, \mathrm{df}=1(P=0.10), I^{2}=63.9 \%$} \\
\hline
\end{tabular}

FIGURE 4: Meta-analysis results of complete resection rate between the two groups.

TABLE 3: Network meta-analysis comparing different classes of injection solutions.

\begin{tabular}{|c|c|c|c|c|}
\hline \multirow{2}{*}{ Interventions } & \multicolumn{2}{|c|}{ En bloc resection } & \multicolumn{2}{|c|}{ Complete resection } \\
\hline & Odds ratios $(95 \% \mathrm{CI})$ & GRADE & Odds ratios $(95 \% \mathrm{CI})$ & GRADE \\
\hline \multicolumn{5}{|l|}{ Fibrinogen mixture } \\
\hline Dextrose & $0.18(0.04,0.89)$ & High & $0.73(0.11,4.96)$ & Moderate \\
\hline Hyaluronic acid & $0.27(0.06,1.21)$ & Moderate & $0.63(0.15,2.60)$ & Moderate \\
\hline Succinylated gelatin & $0.23(0.04,1.27)$ & Moderate & $1.41(0.21,9.63)$ & Moderate \\
\hline Hydroxyethyl starch & $0.43(0.07,2.84)$ & Moderate & $1.44(0.06,31.99)$ & Low \\
\hline Mesna & NA & NA & $0.48(0.02,15.42)$ & Low \\
\hline \multicolumn{5}{|l|}{ Dextrose } \\
\hline Hyaluronic acid & $1.47(0.47,4.59)$ & Moderate & $0.85(0.18,3.96)$ & Moderate \\
\hline Succinylated gelatin & $1.26(0.31,5.11)$ & Moderate & $1.93(0.25,15.08)$ & Low \\
\hline Hydroxyethyl starch & $2.39(0.48,11.94)$ & Low & $1.97(0.08,47.96)$ & Very low \\
\hline Mesna & NA & NA & $0.66(0.02,22.72)$ & Low \\
\hline \multicolumn{5}{|l|}{ Hyaluronic acid } \\
\hline Succinylated gelatin & $0.85(0.23,3.19)$ & Moderate & $2.26(0.49,10.52)$ & Low \\
\hline Hydroxyethyl starch & $1.62(0.35,7.52)$ & Moderate & $2.31(0.13,41.29)$ & Very low \\
\hline Mesna & NA & NA & $0.77(0.03,20.39)$ & Low \\
\hline \multicolumn{5}{|l|}{ Succinylated gelatin } \\
\hline Hydroxyethyl starch & $1.90(0.33,10.81)$ & Low & $1.02(0.04,24.72)$ & Low \\
\hline Mesna & NA & NA & $0.34(0.01,11.56)$ & Low \\
\hline \multicolumn{5}{|l|}{ Hydroxyethyl starch } \\
\hline Mesna & NA & NA & $0.33(0.00,27.72)$ & Very low \\
\hline
\end{tabular}

GRADE: Grading of Recommendations, Assessment, Development, and Evaluation. 


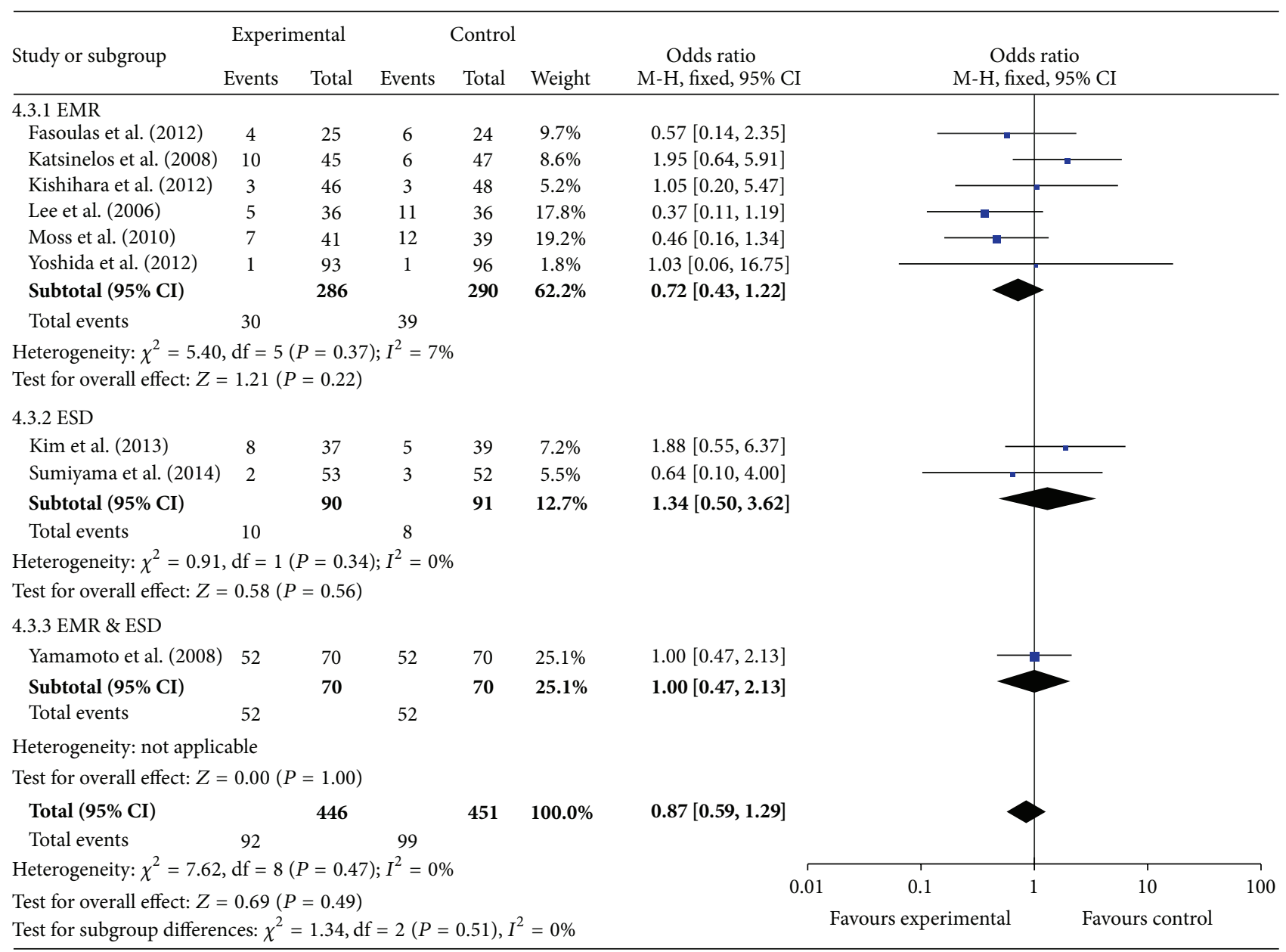

FIGURE 5: Subgroup meta-analysis of total complications.

operation time and increased complication incidence [22]. Therefore, the different method used in the trials would not influence the main outcome measure in the study. When the lesion size was large, it also had high possibilities to be diagnosed as carcinoma and invade into deep tissue [23, 24]; thus the difficulty of operation was really increased. Except one study reporting the average lesion size was larger than $20 \mathrm{~mm}$, other studies all included patients with average lesion size smaller than $20 \mathrm{~mm}$, and this difference may induce a lower en bloc resection rate and complete resection rate, which may be a source of heterogeneity. Meanwhile, lesion location may also have had some impacts on the results $[10,24]$, while we did not find any obvious difference between the studies as shown in Table 1.

When a lesion size is smaller than $20 \mathrm{~mm}$, the required time for operation is short; although NS with low molecular weight is easy to diffuse to surrounding tissues, it can maintain enough mucosal elevation height and duration for operation [16], so this may explain the little difference between the other injection solutions and NS in terms of clinical outcomes for EMR. However, for lesion size larger than $20-30 \mathrm{~mm}$, the required time is relatively long. Endoscopic operator sometimes needs to administer an additional injection when NS was used as submucosal injection solution; in this case the other injection solutions also had advantages in terms of operation time and additional injection [15], and all these terms are important for not only difficult operations but also nonexpert endoscopic operators.

So compared with NS, other newly developed submucosal injection solutions really improved the clinical outcomes. Because the pooled analysis results only reflected an overall effect of the other injection solutions, we further defined the separate effect of each injection solution to explore the more effective ones though network meta-analysis method. According to the type of injection solution, we divided all of them into 6 subgroups including fibrinogen mixture (FM), dextrose (DE), sodium hyaluronic acid (SHA), succinylated gelatin (SG), hydroxyethyl starch (HES), and mesna (ME). In aspect of en bloc resection, dextrose was significantly better than FM, and the other solutions also seemed to be better than FM, while all of them failed to reach statistical significance. And it seemed that dextrose also achieved a high en bloc resection rate compared to SHA, SG, and HES, while no significant difference was found. In aspect of complete resection, SHA and dextrose were both better than the others except for mesna, while it should be suspected that the result 


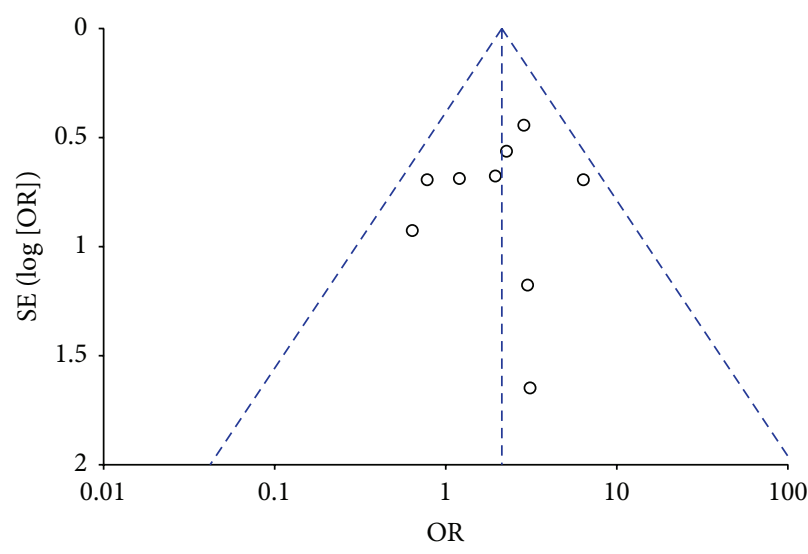

(a)

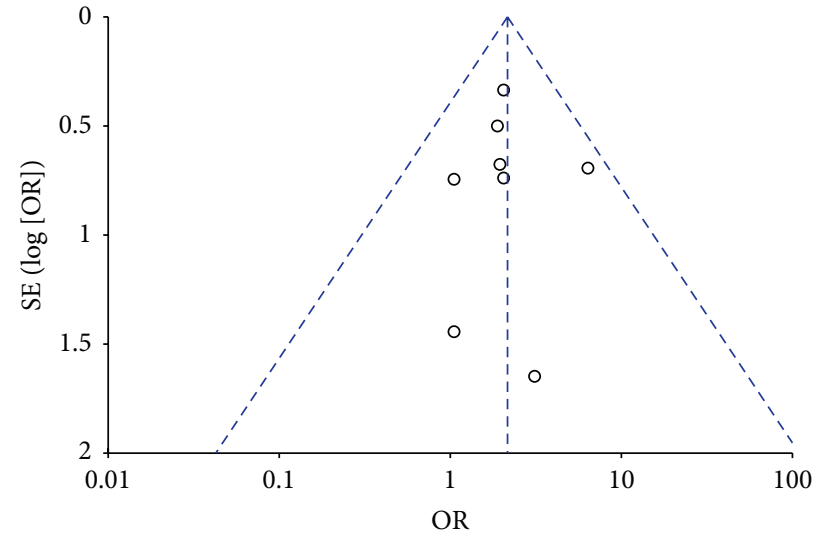

(b)

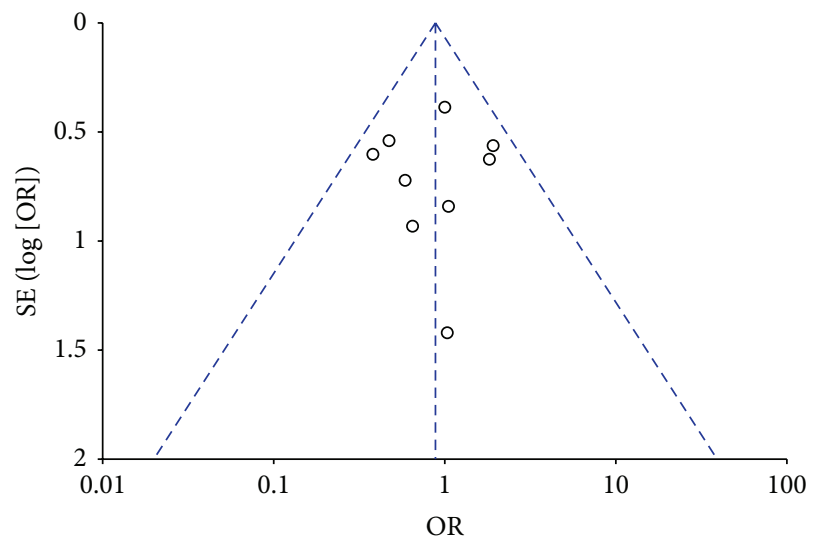

(c)

Figure 6: Funnel plot of publication bias. (a) En bloc resection rate, (b) complete resection rate, and (c) total complications.

of mesna always had a wide 95\% CI. Therefore, current evidence indicated that there was no significant difference between the newly developed injection solutions, and in aspects of en bloc resection rate and complete rate they did not significantly differ from each other. Meanwhile, the abilities of the other solutions to maintain the mucosal elevation seemed to be equivalent as they all declared that no additional injection was needed.

To precisely make a histologic diagnosis, a submucosal injection solution should not damage the cut tissue, and it was reported that hypertonic saline and dextrose caused immediate and delayed porcine tissue damage [7]. In contrast, NS, glycerol, SHA, and FM did not cause any apparent tissue damage, while no relevant studies were performed for SG, HES, and mesna [10]. Except for the fact that dextrose was reported to cause postpolypectomy syndrome more than NS (13\% versus 2\%), it was explained that the dextrose did not cause this itself but was injected too deeply into the muscular colonic wall and obliterated the local vascular supply [12]; FM had a high viscosity of fibrinogen which might conjecture the injection materials [10]; and SHA can to some extent stimulate the growth of residual tumor cells [25]. So SHA is a really ideal injection solution, except for its major disadvantages including high cost, no wide availability, and special storage requirement [26]. The reported price of SHA was $\$ 50-120 / \mathrm{mL}$, FM was $\$ 0.2 / \mathrm{mL}$, glycerol was $\$ 0.01-$ $0.03 / \mathrm{mL}$, SG was $\$ 0.02 / \mathrm{mL}$, and NS was $<\$ 0.01 / \mathrm{mL}$, while the price of HES and mesna was not reported. It was obvious that, compared with SHA, the other injection solutions all significantly reduced finical costs and were easily prepared.

There are still some possible limitations in the current study: (1) although the overall methodological quality is good, two studies have a poor quality with level of C $[14,18]$; (2) as the relative small cases in the analysis, the results of FM and HES should be supported by more studies; (3) endoscopic operator was reported by most studies with performance experience of ESD and EMR > 300 cases, excepted one study that had unexperienced endoscopist [18]; and endoscopic operator's experience and learning curve really significantly influence the outcomes; the current study could not draw a conclusion on this; (4) glycerol was also a promising submucosal injection solution, while the current study could not quantitively analyze it because no relevant RCT was found.

In conclusion, other newly developed submucosal injection solutions significantly increased the en bloc resection rate and complete resection rate and decreased the bleeding rate and finical cost of endoscopic resection in GI tract, while 
current evidence did not find the difference between them, which need to be explored by further studies.

\section{Conflict of Interests}

All the authors state that there was no conflict of interests and that no source of funding existed.

\section{Authors' Contribution}

Zhang Yu Huai designed the research, Zhang Yu Huai, Wei Feng Xian, and Luo Chang Jiang conducted the literature search separately, and Luo Chang Jiang and Wang Xi Chen extracted and cross-checked the data. Data analysis was performed by Zhang Yu Huai and Wei Feng Xian. All the authors took part in the paper preparation and revision.

\section{Acknowledgment}

The authors thank Tian Jin Hui (Evidence-Based Medicine Center of Lanzhou University) for confirming the literature result and data analysis.

\section{References}

[1] Y.-M. Park, E. Cho, H.-Y. Kang, and J.-M. Kim, "The effectiveness and safety of endoscopic submucosal dissection compared with endoscopic mucosal resection for early gastric cancer: a systematic review and metaanalysis," Surgical Endoscopy, vol. 25, no. 8, pp. 2666-2677, 2011.

[2] J. K. A. Jameel, S. H. Pillinger, P. Moncur, H. H. Tsai, and G. S. Duthie, "Endoscopic mucosal resection (EMR) in the management of large colo-rectal polyps," Colorectal Disease, vol. 8, no. 6, pp. 497-500, 2006.

[3] H. Ono, H. Kondo, T. Gotoda et al., "Endoscopic mucosal resection for treatment of early gastric cancer," Gut, vol. 48, no. 2, pp. 225-229, 2001.

[4] H. Y. Jung, "Extended approach of EMR/ESD in stomach cancer: CON," Journal of the Korean Gastric Cancer Association, vol. 8 , no. 1 , pp. $5-8,2008$.

[5] K. Nonaka, M. Nishimura, S. Yoshinaga et al., "Comparison of endoscopic submucosal dissection using sodium hyaluronate and needle-knife versus endoscopic mucosal resection for colonic laterally spreading tumors," Gastrointestinal Endoscopy, vol. 67, no. 5, p. AB282, 2008.

[6] T. Uraoka, Y. Saito, K. Yamamoto, and T. Fujii, "Submucosal injection solution for gastrointestinal tract endoscopic mucosal resection and endoscopic submucosal dissection," Drug Design, Development and Therapy, no. 2, pp. 131-138, 2008.

[7] M. Fujishiro, N. Yahagi, K. Kashimura et al., "Tissue damage of different submucosal injection solutions for EMR," Gastrointestinal Endoscopy, vol. 62, no. 6, pp. 933-942, 2005.

[8] J. P. T. Higgins and S. Green, Handbook for Systematic Reviews of Interventions Version 5.0.1, 2008, http://www.cochrane-handbook.org/.

[9] Y.-W. Zhang, Y.-L. Zhang, H. Pan, F.-X. Wei, Y.-C. Zhang, and Y. Shao, "Chemotherapy for patients with gastric cancer after complete resection: a network meta-analysis," World Journal of Gastroenterology, vol. 20, p. 584, 2014.
[10] S.-H. Lee, J.-H. Park, D. H. Park et al., "Clinical efficacy of EMR with submucosal injection of a fibrinogen mixture: a prospective randomized trial," Gastrointestinal Endoscopy, vol. 64, no. 5, pp. 691-696, 2006.

[11] D. P. Hurlstone, K. I. Fu, S. R. Brown et al., "EMR using dextrose solution versus sodium hyaluronate for colorectal Paris type I and 0-II lesions: a randomized endoscopist-blinded study," Endoscopy, vol. 40, no. 2, pp. 110-114, 2008.

[12] P. Katsinelos, J. Kountouras, G. Paroutoglou et al., "A comparative study of $50 \%$ dextrose and normal saline solution on their ability to create submucosal fluid cushions for endoscopic resection of sessile rectosigmoid polyps," Gastrointestinal Endoscopy, vol. 68, no. 4, pp. 692-698, 2008.

[13] H. Yamamoto, N. Yahagi, T. Oyama et al., "Usefulness and safety of $0.4 \%$ sodium hyaluronate solution as a submucosal fluid 'cushion' in endoscopic resection for gastric neoplasms: a prospective multicenter trial," Gastrointestinal Endoscopy, vol. 67, no. 6, pp. 830-839, 2008.

[14] T. Kizu, N. Uedo, R. Chatani et al., "A prospective randomized controlled trial comparing ' $0.4 \%$ sodium hyaluronate' versus 'normal saline solution' for endoscopic submucosal dissection in gastric neoplasia by supervised residents," Gastrointestinal Endoscopy, vol. 71, no. 5, pp. AB104-AB105, 2010.

[15] A. Moss, M. J. Bourke, and A. J. Metz, "A randomized, doubleblind trial of succinylated gelatin submucosal injection for endoscopic resection of large sessile polyps of the colon," The American Journal of Gastroenterology, vol. 105, no. 11, pp. 23752382, 2010.

[16] K. Fasoulas, G. Lazaraki, G. Chatzimavroudis et al., "Endoscopic mucosal resection of giant laterally spreading tumors with submucosal injection of hydroxyethyl starch: comparative study with normal saline solution," Surgical Laparoscopy, Endoscopy \& Percutaneous Techniques, vol. 22, no. 3, pp. 272278, 2012.

[17] T. Kishihara, A. Chino, N. Uragami et al., "Usefulness of sodium hyaluronate solution in colorectal endoscopic mucosal resection," Digestive Endoscopy, vol. 24, no. 5, pp. 348-352, 2012.

[18] N. Yoshida, Y. Naito, Y. Inada et al., "Endoscopic mucosal resection with $0.13 \%$ hyaluronic acid solution for colorectal polyps less than $20 \mathrm{~mm}$ : a randomized controlled trial," Journal of Gastroenterology and Hepatology, vol. 27, no. 8, pp. 1377-1383, 2012.

[19] Y. D. Kim, J. Lee, J. Y. Cho et al., "Efficacy and safety of 0.4 percent sodium hyaluronate for endoscopic submucosal dissection of gastric neoplasms," World Journal of Gastroenterology, vol. 19, no. 20, pp. 3069-3076, 2013.

[20] K. Sumiyama, H. Toyoizumi, T. R. Ohya et al., "A doubleblind, block-randomized, placebo-controlled trial to identify the chemical assistance effect of mesna submucosal injection for gastric endoscopic submucosal dissection," Gastrointestinal Endoscopy, vol. 79, no. 5, pp. 756-764, 2014.

[21] B.-H. Min, J. H. Lee, J. J. Kim et al., "Clinical outcomes of endoscopic submucosal dissection (ESD) for treating early gastric cancer: comparison with endoscopic mucosal resection after circumferential precutting (EMR-P)," Digestive and Liver Disease, vol. 41, no. 3, pp. 201-209, 2009.

[22] Y. Cao, C. Liao, A. Tan, Y. Gao, Z. Mo, and F. Gao, "Metaanalysis of endoscopic submucosal dissection versus endoscopic mucosal resection for tumors of the gastrointestinal tract," Endoscopy, vol. 41, no. 9, pp. 751-757, 2009.

[23] N. Kobayashi, Y. Saito, Y. Sano et al., "Determining the treatment strategy for colorectal neoplastic lesions: endoscopic 
assessment or the non-lifting sign for diagnosing invasion depth?" Endoscopy, vol. 39, no. 8, pp. 701-705, 2007.

[24] S.-H. Lee, W.-Y. Cho, H.-J. Kim et al., "A new method of EMR: Submucosal injection of a fibrinogen mixture," Gastrointestinal Endoscopy, vol. 59, no. 2, pp. 220-224, 2004.

[25] Y. Matsui, M. Inomata, K. Izumi, K. Sonoda, N. Shiraishi, and S. Kitano, "Hyaluronic acid stimulates tumor-cell proliferation at wound sites," Gastrointestinal Endoscopy, vol. 60, no. 4, pp. 539-543, 2004.

[26] J. J. Hyun, R. C. Hwang, J. C. Hoon et al., "Comparison of the characteristics of submucosal injection solutions used in endoscopic mucosal resection," Scandinavian Journal of Gastroenterology, vol. 41, no. 4, pp. 488-492, 2006. 


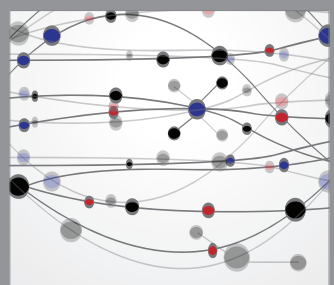

The Scientific World Journal
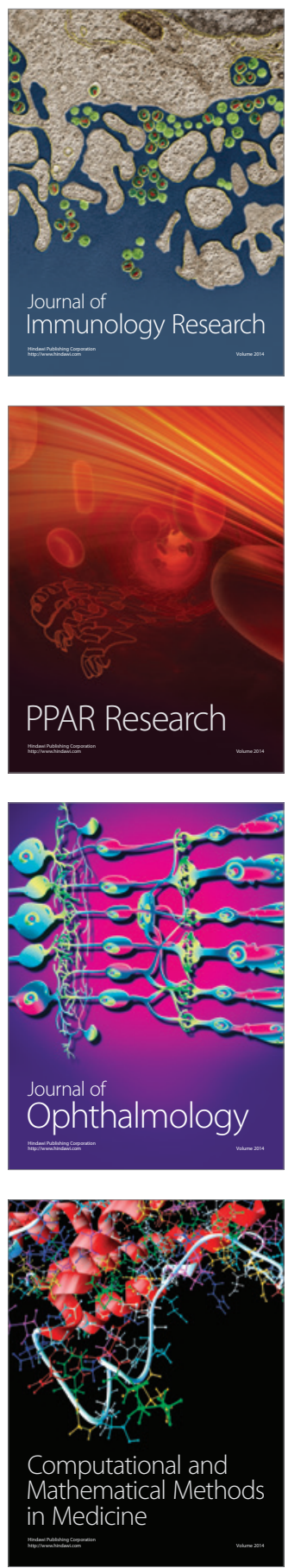

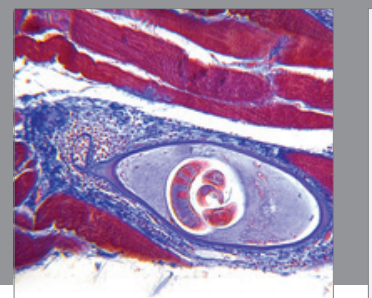

Gastroenterology

Research and Practice
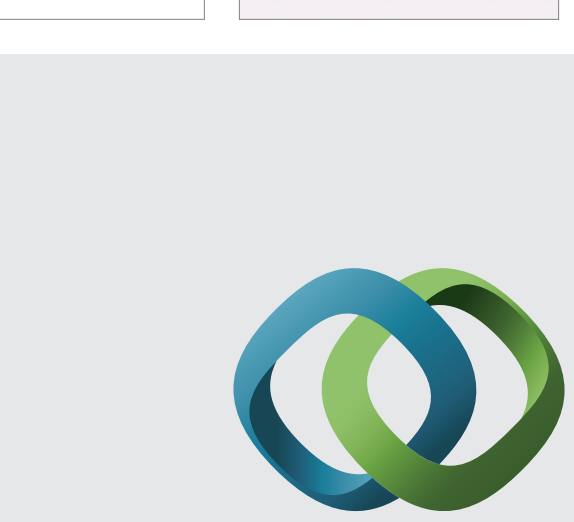

\section{Hindawi}

Submit your manuscripts at

http://www.hindawi.com
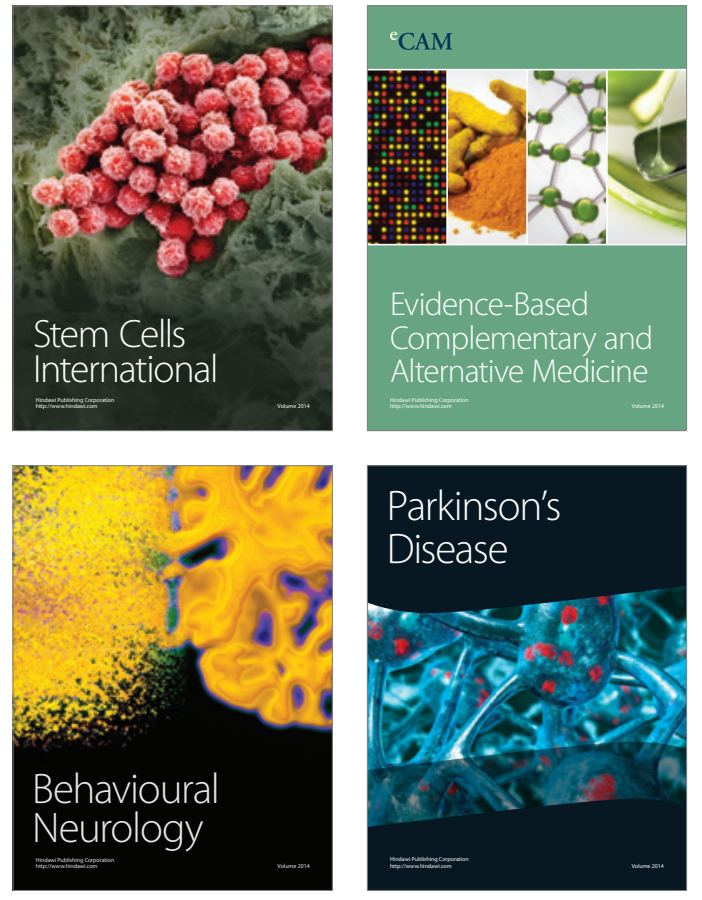
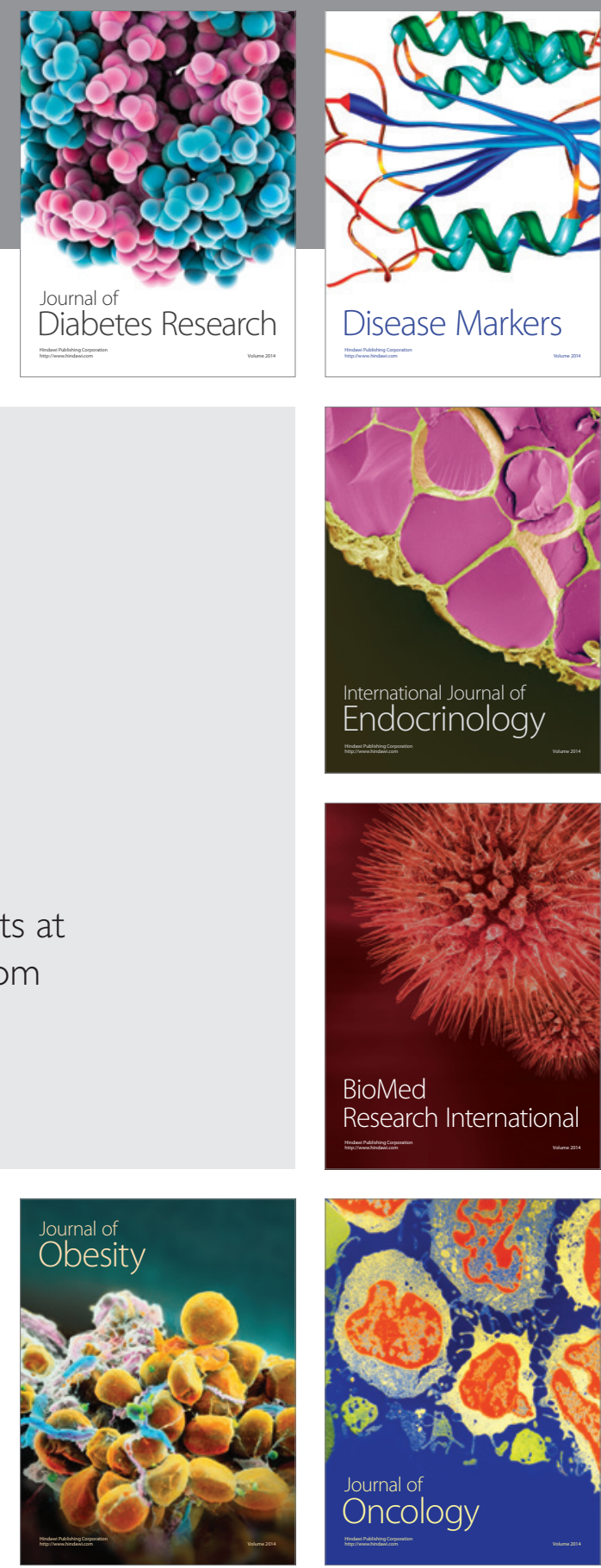

Disease Markers
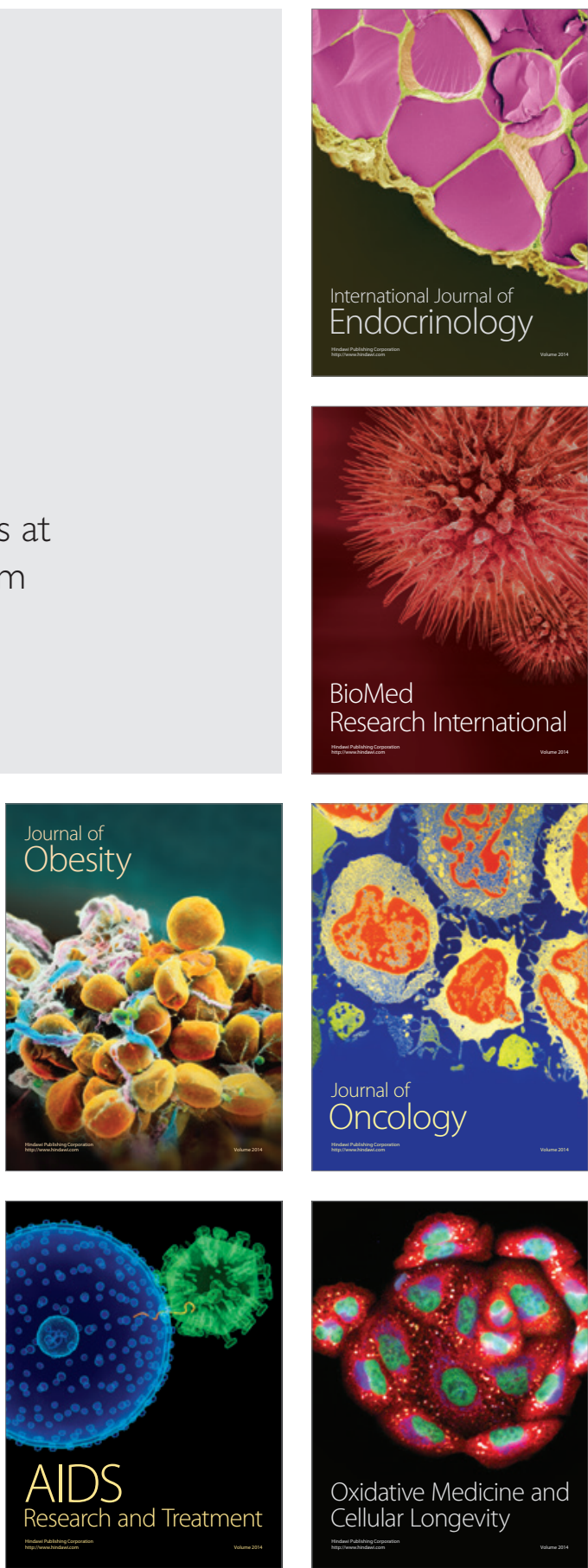